\title{
IDENTIFICAÇÃO DE LITOTIPOS NOS PERFIS DE POÇO DO CAMPO DE NAMORADO (BACIA DE CAMPOS, BRASIL) E DO LAGO MARACAIBO (VENEZUELA) APLICANDO ESTATÍSTICA MULTIVARIADA
}

\author{
ROOSEVELT TAVARES FLEXA ${ }^{1}$, ANDRÉ ANDRADE ${ }^{2} \&$ ABEL CARRASQUILLA ${ }^{1}$
}

\begin{abstract}
IDENTIFICATION OF LITHOTYPES IN WELL LOGS OF NAMORADO FIELD (CAMPOS BASIN, BRAZIL) AND MARACAIBO LAKE (VENEZUELA) APPLYING MULTIVARIED STATISTICS The identification of lithotypes along a borehole is one of the most important contributions of borehole geophysics to geological studies inside the petroleum industry. However, it is a difficult task, because the measurements performed in the well express the changes of the physical properties and, not necessarily, the lithological variations. This work shows a methodology based on discriminant analysis technique for the identification of lithotypes in well logs, by means of the association between the physical property variations and the lithological types in depth. The geophysical logs applied for the evaluation of this methodology were gamma ray $(G R)$, sonic $(D T)$, neutronic porosity $(P H I N)$, density $(R H O B)$ and resistivity $(I L D)$. In this form, we tested this scheme in two wells of Namorado Field (Campos Basin, Brazil) and another two of Maracaibo Lake (Venezuela). The obtained results are interesting in the sense of the automatic identification of lithotypes using well logs. Thus, the optained results allow to observe an excellent agreement between the well log interpretations or the geological column descriptions of the well and the lithological types identified by the method.
\end{abstract}

Keywords: discriminant analysis, geophysical logging, Campos Basin, Maracaibo Lake.

\begin{abstract}
Resumo A identificação de litotipos ao longo de um poço é uma das contribuições mais importantes da geofísica aos estudos geológicos dentro da indústria de petróleo. Entretanto, é uma das tarefas mais difíceis, porque as medidas executadas no poço expressam as mudanças das propriedades físicas e, não necessariamente, as variações litológicas. Este trabalho mostra uma metodologia baseada na técnica da análise discriminante para a identificação de litotipos nos perfis de poços, onde procuramos a associação entre as variações das propriedades físicas e os tipos litológicos em profundidade. Os perfis geofísicos utilizados para a avaliação desta metodologia são raios gama $(G R)$, sônico $(D T)$, porosidade neutrônica $(P H I N)$, densidade $(R H O B)$ e resistividade $(I L D)$. Desta forma, testamos o esquema desenvolvido em dois poços do Campo de Namorado (Bacia de Campos, Brasil) e em outros dois do Lago Maracaibo (Venezuela). Os resultados obtidos são interessantes no sentido da identificação automática de litotipos usando perfis de poço. Assim, os resultados obtidos permite um excelente acordo entre as interpretações dos perfis ou as colunas estratigráficas dos poços e os tipos litológicos identificados pelo método.
\end{abstract}

Palavras-chave: análise discriminante, geofísica de poço, Bacia de Campos, Lago Maracaibo.

INTRODUÇÃO Atualmente, muitos estudos mostram uma boa aplicabilidade das técnicas da análise estatística multivariada no processamento e interpretação de dados geofísicos (Couto 1994). No caso da geofísica de poço, uma das aplicações freqüentemente apresentada trata da identificação e da separação das eletrofácies e a sua associação às diversos litotipos atravessados pelo poço. Outros estudos, onde também se têm aplicado a estatística multivariada com sucesso, se referem a problemas da engenharia de reservatório (Nitters et al. 1995).

Neste trabalho apresenta-se a aplicação da análise discriminante a dados de perfis geofísicos de dois poços do Campo de Namorado na Bacia de Campos, localizado na costa norte do Rio de Janeiro (Fig. 1), e, seguidamente, nossa metodologia foi também aplicada aos perfis de dois poços do Lago Maracaibo, no oeste da República de Venezuela (Fig. 2).

A Bacia de Campos possui destacada importância econômica, uma vez que é responsável por mais $75 \%$ da produção brasileira de petróleo e $80 \%$ das reservas brasileiras exploráveis (Guardado et al. 2000). Como outras bacias da costa leste brasileira, a Bacia de Campos é uma típica bacia de margem divergente, tendo sua origem relacionada ao rompimento do supercontinente Gondwana e à consequiente formação do Oceano Attântico. Nesta bacia, a interligação das rochas geradoras e das rochas reservatórios pósevaporíticas está relacionada à tectônica profunda associada à fase rift e à movimentação adiastrófica associada à halocinese. A migração do hidrocarboneto é facilitada pela criação de "janelas" nos evaporitos. As principais rochas geradoras da Bacia de Campos estão na Formação Lagoa Feia, quanto que, suas principais acumulações de hidrocarbonetos acontecem nos basaltos da Formação Cabiúnas, nas coquinas da Formação Lagoa Feia, nos carbonatos porosos da Formação Macaé e nos arenitos turbidíticos da Formação Campos, sendo estes últimos os maiores reservatórios de petróleo da Bacia de Campos (Dias et al. 1990). O Membro Outeiro da Formação Macaé reúne, além de calcilutito creme, marga cinza-clara e folhelhos cinza, arenitos turbiditos informalmente conhecidos como arenitos Namorado, que por vezes ocorrem em camadas isoladas ou confinados em calhas deposicionais (Fig. 3), as quais subsidiram diferencialmente em resposta à halocinese

1 - Laboratório de Engenharia e Exploração de Petróleo, CCT, UENF, Rod. Amaral Peixoto Km 163, Av. Brenand S/N, CEP 27925-031, Macaé - RJ Brasil, email: rtflexa@lenep.uenf.br, abel@lenep.uenf.br

2 - Departamento de Geofísica, CG, Universidade Federal do Pará, Campus Universitário do Guamá, Rua Augusto Corrêa, 01, CEP 66075-110, Belém - PA - Brasil email: aandrade@ufpa.br 


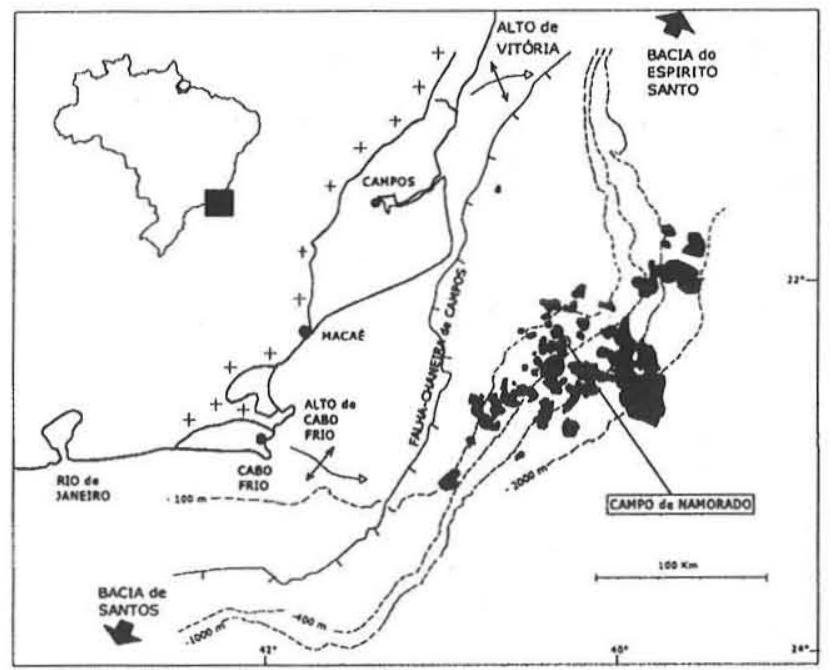

Figura I - Mapa de Localização da Bacia de Campos no Estado do Rio de Janeiro, Brasil.

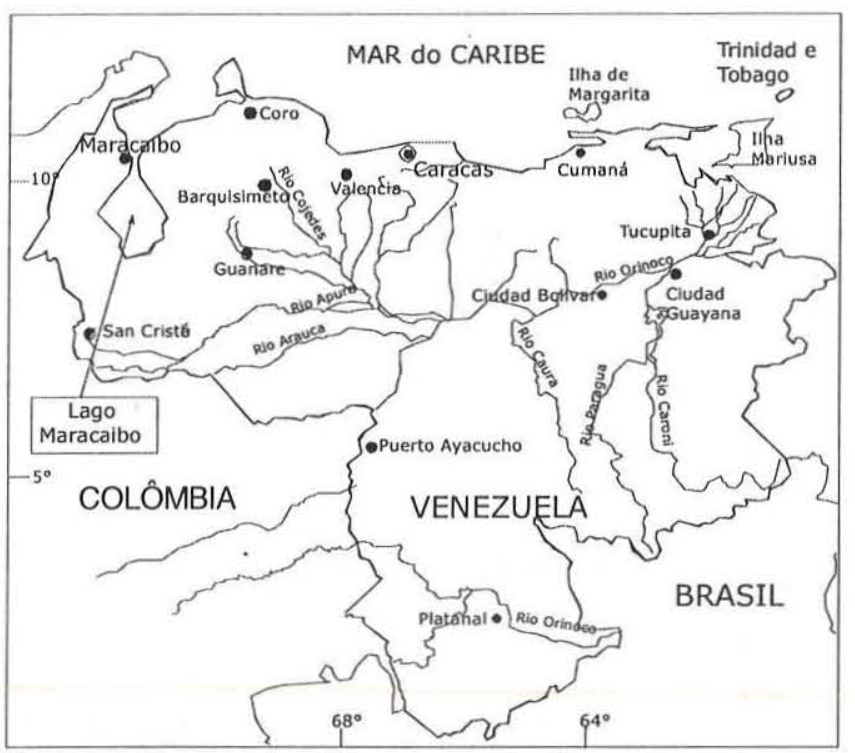

Figura 2 - Mapa de Localização do Lago Maracaibo no oeste da República da Venezuela.

\section{(Rangel et al. 1994).}

Nos últimos anos, foram disponibilizados, pela ANP (Agência Nacional de Petróleo), dados referentes ao Campo de Namorado. Assim, os dados de perfis de poço e os da análise seqüencial de testemunho deste campo foram utilizados no desenvolvimento deste trabalho.

Por outro lado, no caso do Lago Maracaibo, a evolução geológica desta bacia apresenta características relevantes para o desenvolvimento de sistemas petrolíferos, incluindo a sua tectônica e a sua sedimentação do Holoceno. Entre as formações geológicas presentes, a Lagunillas ganha destaque, a qual se originou no baixo-meio Mioceno e consiste de arenito, folhelho e carvão, depositados em ambientes deltaico e fluvial (Talukdar \& Marcano 1994). No caso desta bacia, os dados de perfis de poço e suas

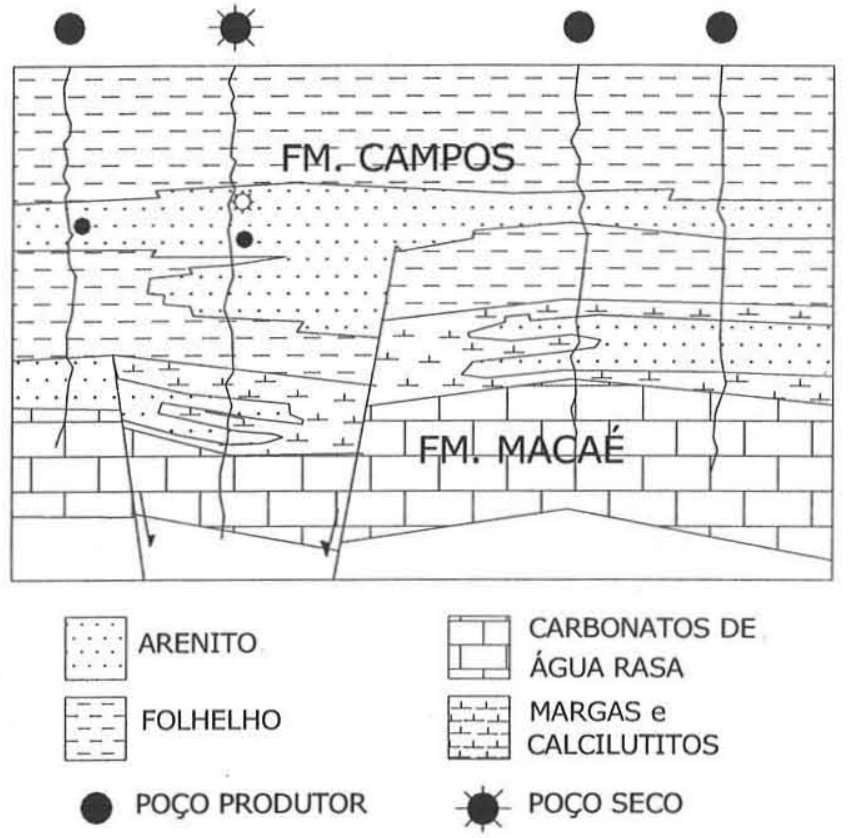

Figura 3 - Arenitos Namorado pertencente ao Membro Outeiro da Formação Macaé.

respectivas interpretações foram também incluídos no desenvolvimento deste trabalho.

MÉTODOS Existem diversas técnicas da estatística multivariada, que podem ser aplicadas na solução de uma enorme variedade de problemas, nas várias áreas do conhecimento, como na geofísica, geoquímica, geografia, estatística e outras. Uma destas técnicas é a análise discriminante, que tem grande eficiência na solução de determinados problemas geofísicos, quando não se dispõe de amostras de rochas (Serra \& Abbot 1989).

A análise discriminante é utilizada nos casos em que se deseja realizar a separação estatística entre dois ou mais grupos de dados, que devem ser definidos previamente pela caracterização de suas variáveis discriminantes. Essas variáveis são combinadas linearmente para que seja acentuada a diferença entre os grupos, gerando, assim, os coeficientes empregados na solução da função discriminante, resultando, portanto, em apenas um único índice discriminante, que representa a posição da amostra ao longo da reta definida pela função discriminante (Bucheb 1991).

A técnica da análise discriminante consiste em encontrar uma transformação linear que resulte na razão de mínima diferença entre um par de grupos com médias multivariadas e uma variância multivariada dentro de cada par de grupos. Considerando-se o. caso de dois grupos, como consistindo de dois conjuntos em um espaço multivariado, deve-se buscar uma orientação junto a qual ambos conjuntos tenham a maior separação, enquanto, simultaneamente, cada conjunto tenha uma mínima variação. Isto é mostrado ilustrativamente para o caso bidimensional (bivariado), na figura 4. Como observa-se na figura, uma separação entre os grupos $A$ e $B$ não pode ser obtida usando as variáveis $X_{1}$ e $X_{2}$. No entanto, é possível encontrar uma nova orientação junto a qual os dois conjuntos são separados o máximo possível e tendo uma mínima variação em cada grupo (Flexa 2001).

Assim, a análise discriminante tenta pesar e combinar linearmente as variáveis discriminantes, de modo a maximizar as 


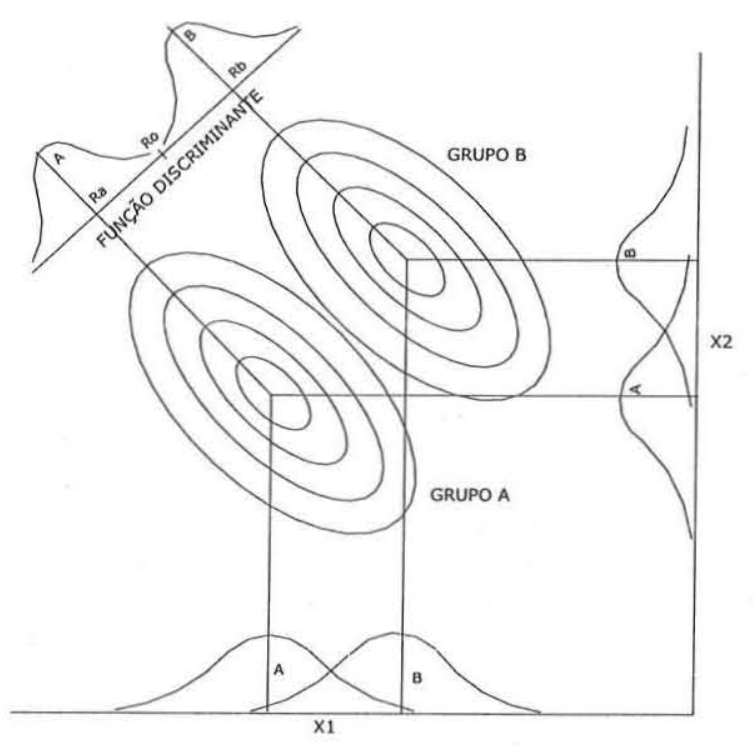

Figura 4 - Representação da função discriminante aplicada a um exemplo de distribuição bivariada (modificado de Davis 1973).

diferenças estatísticas entre os diversos grupos. As funções que discriminam os grupos entre si denominam-se função discriminante, e são matematicamente definidas na forma:

ou

$$
R=\lambda_{1} \psi_{1}+\lambda_{2} \psi_{2}+\ldots+\lambda_{m} \psi_{m}
$$

$$
R=\sum_{k=1}^{m} \lambda_{k} \psi_{k}
$$

onde $R$ é o índice discriminante da função, $\lambda_{k}$ são os coeficientes da função discriminante e $\psi_{k}$ os valores da k-ésima variável de um determinado objeto, com:

$$
1 \leq k \leq m
$$

em que $m$ é o número de variáveis utilizado na análise.

Um método que pode ser usado para encontrar os coeficientes da função discriminante, segundo Davis (1973), de modo que a probabilidade de classificar erroneamente um novo elemento em qualquer um dos grupos seja mínima, é usar a regressão múltipla onde a variável dependente consiste na diferença das médias multivariadas de dois grupos. Isto se traduz por uma equação matricial dada por:

$$
\left\lfloor S_{C}^{2}\right\rfloor \cdot[\lambda]=[d]
$$

na qual $\left\lfloor s_{C}^{2}\right\rfloor$ é uma matriz $m \times m$, denominada de matriz das variâncias e covariâncias combinadas de $m$ variáveis. $\mathrm{O}$ vetor coluna $[\lambda]$ é o vetor formado pelos coeficientes da função discriminante, e o vetor coluna $[d]$, representa um vetor formado pelas diferenças entre as médias dos dois grupos.

A equação (2) pode ser resolvida usando os conceitos da inversão e multiplicação de matrizes. Assim:

$$
\left[s_{C}^{2}\right]^{-1} \cdot\left[s_{C}^{2}\right] \cdot[\lambda]=\left[s_{C}^{2}\right]^{-1} \cdot[d]
$$

considerando que $\left[s_{C}\right]^{-1}$ exista e como $\left[s_{c}^{2}\right]^{-1},\left[s_{c}^{2}\right]=\left[I_{m}\right]$ onde $I_{m}$ é a matriz identidade, temos:

$$
\begin{aligned}
& \quad\left[I_{m}\right] \cdot[\lambda]=\left[S_{C}^{2}\right]^{-1} \cdot[d] \\
& \text { ou, }[d]=\left[S_{C}^{2}\right]^{-1} \cdot[d]
\end{aligned}
$$

Para calcular os coeficientes da função discriminante devemos determinar as entradas na equação matricial na Equação (4). O vetor coluna $[d]$ pode ser, simplesmente, encontrado por:

$$
d_{j}=\bar{A}_{j}-\bar{B}_{j}=\frac{\sum_{i=1}^{n_{a}} A_{i j}}{n_{a}}-\frac{\sum_{i=1}^{n_{b}} B_{i j}}{n_{b}}
$$

Nesta notação, $A_{i j}$ é i-ésima observação da variável $j$ no grupo $A$; $\bar{A}$ é a média da variável $j$ no grupo. Essas mesmas convenções são aplicadas ao grupo $B$. Portanto, as médias multivariadas dos grupos $A$ e $B$ podem ser consideradas como formando dois vetores, da seguinte forma compacta,

ou, na forma expandida,

$$
\left[\begin{array}{c}
d_{1} \\
d_{2} \\
\cdot \\
\cdot \\
d_{m}
\end{array}\right]=\left[\begin{array}{c}
\overline{A_{1}} \\
\overline{A_{2}} \\
\cdot \\
\dot{A_{m}}
\end{array}\right]-\left[\begin{array}{c}
\overline{B_{1}} \\
\overline{B_{2}} \\
\cdot \\
\overline{B_{m}}
\end{array}\right]
$$

Para construir a matriz das variâncias e covariâncias combinadas, devemos calcular uma matriz das somas dos quadrados e os produtos cruzados de todas as variáveis no grupo $A(S C A)$ e uma matriz semelhante para a grupo $B(S C B)$.

Consideremos, por exemplo, o grupo $A$ :

$$
S C A_{j k}=\sum_{i=1}^{n_{a}}\left(A_{i j} \cdot A_{i k}\right)-\frac{\sum_{i=1}^{n_{a}} A_{i j} \sum_{i=1}^{n_{4}} A_{i k}}{n_{a}}
$$

onde $A_{i j}$ representa a i-ésima observação da variável $j$ no grupo $A$, e $A_{i k}$ representa a i-ésima observação da variável $k$ no mesmo grupo. Naturalmente, esta quantidade será a soma dos quadrados da variável $k$ quando $j=k$. Semelhantemente, uma matriz de somas dos quadrados e produtos cruzados pode ser encontrada para o grupo $B$, ou seja,

$$
S C B_{j k}=\sum_{i=1}^{n_{b}}\left(B_{i j} \cdot B_{i k}\right)-\frac{\sum_{i=1}^{n_{b}} B_{i j} \sum_{i=1}^{n_{b}} B_{i k}}{n_{b}}
$$

A matriz das variâncias e covariâncias pode agora ser calculada como:

$$
\left[S_{C}^{2}\right]=\frac{[S C A]+[S C B]}{n_{a}+n_{b}-2}
$$

Desta forma, temos todas os termos de entradas necessários para resolver a Equação (4), obtendo-se os $\lambda$ 's , que são os coeficientes de entrada na equação da função discriminante (1). Com o conjunto de $\lambda$ 's podemos substitui-los na Equação (1), para formarmos a equação discriminante, ou seja, montar a equação linear, que produzirá um único número, o índice discriminante.

A substituição do ponto médio entre os dois grupos na Equação (1), produzirá o índice discriminante $R_{0}$, isto é, para cada valor de $\psi$, na equação (1) inserimos os termos:

Assim obtemos:

$$
\bar{\psi}_{j}=\frac{\bar{A}_{j}+\bar{B}_{j}}{2}
$$

$$
R_{0}=\lambda_{1} \bar{\psi}_{1}+\lambda_{2} \bar{\psi}_{2}+\ldots+\lambda_{m} \bar{\psi}_{m}
$$

A seguir, podemos substituir a média multivariada do grupo na Equação (1) para obter $R_{A}$ (isto é, fazendo $\psi-\bar{A}$.) e a média do grupo $B$ para obter $R_{B}(\Psi=-\bar{B})$. Estes valores $\left(R_{A}\right.$ e $\left.R_{B}\right)$ definem os centros dos dois grupos originais ao longo da função discriminante.

Para o grupo $A$ :

$$
R_{\Lambda}=\lambda_{1} \overline{A_{1}}+\lambda_{2} \bar{A}_{2}+\ldots+\lambda_{m} \bar{A}_{m}
$$


e para o grupo $B$ :

$$
R_{B}=\lambda_{1} \bar{B}_{1}+\lambda_{2} \bar{B}_{2}+\ldots+\lambda_{m} \bar{B}_{m}
$$

Podemos calcular a distância entre as duas médias multivariadas pela simples subtração de $R_{A}-R_{B}$. Isto é equivalente a substituição do vetor de diferenças entre os dois grupos na equação da função discriminante, tomando $\psi=d_{t}$. Assim,

$$
D^{2}=\lambda, d,+\lambda, d+\ldots+\lambda, d
$$

Esta medida de distância é denominada de distância de Mahalanobis ou distância generalizada $D^{2}$, a qual é a separação entre as duas médias multivariadas expressas em unidade de variância combinada.

Podemos concluir que, se as médias dos grupos $A$ e $B$ forem próximas, será difícil distinguí-los, especialmente se ambos possuírem altos valores de variâncias. Por outro lado, se a diferença entre as duas médias for expressiva e as suas variâncias pequenas, a discriminação entre os dois conjuntos será mais efetiva.

A distância de Mahalanobis ajuda no cálculo da contribuição relativa de cada variável $j$ e é dada na forma:

$$
e_{j}=\frac{\lambda_{j} d_{j}}{D^{2}} \cdot 100 \%
$$

em que $d_{j}$ é a diferença entre as j-ésimas médias dos dois grupos.

No nosso trabalho fazemos uso do método descrito para a identificação de litotipos nos perfis de poço, sem sua completa interpretação. Desta forma, buscou-se fazer a associação das variações das propriedades físicas dos litotipos em profundidade, com o emprego de dados de entrada de curvas de perfis geofísicos, como raios gama $(G R)$, tempo de trânsito $(D T)$, porosidade neutrônica $(P H I N)$, densidade (RHOP) e resistividade (ILD), os quais são mostrados na figura 5, para o Poço NA04 do Campo de Namorado, e, na figura 6, para o Poço 1112 do Lago Maracaibo.

A análise discriminante, na forma adotada neste trabalho, trata apenas com distribuições bivariadas. Desta forma, baseados nos princípios teóricos da análise discriminante desenvolvido por Davis (1973), desenvolveu-se um programa capaz de discriminar dois litotipos previamente definidos. Em que pese a existência de um grande número de variáveis envolvidas, optou-se pelo desmembramento da interpretação estatística dos perfis geofísicos em dois problemas bivariados, para o caso dos dados do Campo

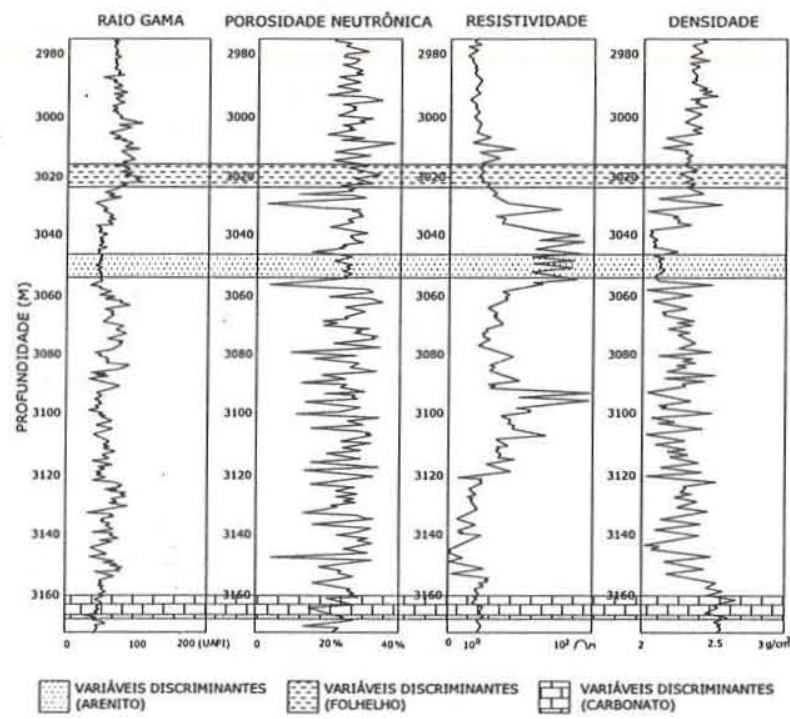

Figura 5 - Perfis utilizados na escolha das variáveis discriminantes para o Poço NAO2 do Campo de Namorado. de Namorado, e de somente um, no caso dos dados do Lago Maracaibo. O primeiro trata da identificação dos tipos litológicos ou separação entre selantes (folhelhos) e reservatórios (arenitos/ carbonatos). E o segundo problema, já com a identificação dos reservatórios, aplicou-se somente a estes a análise discriminante para a identificação entre os tipos litológicos, ou seja, separação entre arenitos e carbonatos. No caso do Lago Maracaibo, esta última etapa não se aplica, devido à ausência de carbonatos na coluna geológica.

A partir de perfis considerados sensíveis ao litotipo, foram construídos bancos de dados, dos quais selecionou-se conjuntos de variáveis discriminantes representativas dos padrões que diferenciam litotipos. O primeiro banco de dados, sensível ao litotipo, foi utilizado para identificar os tipos litológicos selantes (folhelhos) e reservatórios (arenitos/carbonatos). Esse foi útil no caso dos dois campos de petróleo analisados. O outro banco de dados, utilizado somente no Campo de Namorado, também sensível ao litotipo, foi utilizado para identificar arenitos ou carbonatos. É importante enfatizar que, quanto mais discrepantes forem os valores das variáveis discriminantes destes grupos, maior a chance de sucesso da discriminação.

$\mathrm{Na}$ aplicação da análise discriminante adotou-se como referência os Poços NA02 do Campo de Namorado e 1112 do Lago Maracaibo. Sobre os perfis desses poços são interpretados e construídos os bancos de dados que constituirão as funções discriminantes. Considerando a ausência de importantes efeitos diagenéticos, pode-se aplicar estas funções discriminantes' aos poços vizinhos, por exemplo, o Poço NA04 do Campo de Namorado e o Poço 991 do Lago Maracaibo. Deste modo, estende-se a interpretação realizada no poço de referência ao longo de todo o campo petrolífero e, conseqüentemente, a identificação dos tipos litológicos em profundidade.

RESULTADOS A partir da interpretação realizada nos poços de referência, obteve-se funções discriminantes para cada um dos problemas bivariados. Apresentam-se aqui as verificações e as validações das funções discriminantes no poço de referência e em um poço vizinho de cada bacia estudada.

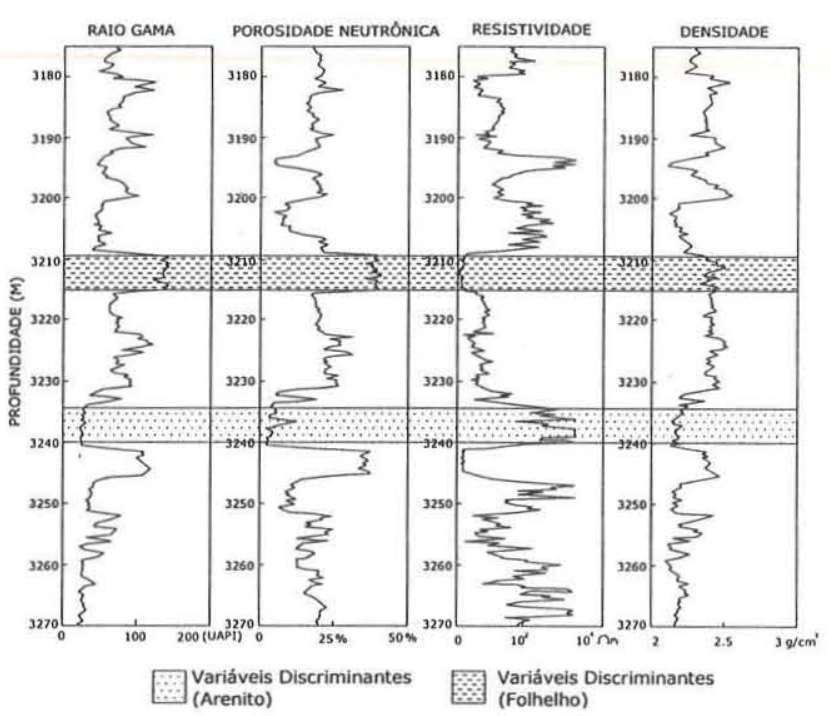

Figura 6 - Perfis utilizados na escolha das variáveis discriminantes para o Poço 1112 do Lago Maracaibo. 
A função discriminante, obtida para a identificação dos tipos litológicos nos dois poços de referência, são mostradas nas Tabelas 1 e 2 (Campo de Namorado e Lago Maracaibo, respectivamente), por meio de seus coeficientes discriminantes para cada tipo de perfil utilizado. Apresentam-se ainda, nessas tabelas, os índices discriminantes, onde $R_{0}$ representa a separação entre os dois litotipos, $\mathrm{R}_{\mathrm{A}}$ é o índice discriminante característico dos reservatórios (arenitos/carbonatos) e $\mathrm{R}_{\mathrm{B}}$ é o índice discriminante característico dos selantes (folhelhos).

O critério de classificação adotado é baseado na posição do índice discriminante médio $\left(\mathrm{R}_{0}\right)$. Calculado o índice discriminante para cada profundidade, este é comparado com o índice discriminante médio. Para cada índice discriminante maior que $\mathrm{R}_{0}$ assume-se o litotipo nessa profundidade como um elemento do grupo dos reservatórios. Para os outros índices menores que $\mathrm{R}_{0}$ assume-se ao litotipo nessa profundidade como elemento do grupo dos selantes (Fig. 7 para o Campo de Namorado e Fig. 8 para o Lago Maracaibo).

A função discriminante, obtida para a identificação dos tipos litológicos nos poços de referência, especificamente aplicada nos trechos de perfil previamente classificados como reservatórios, são mostradas na Tabela 3 (Campo de Namorado) através dos seus coeficientes discriminantes para cada tipo de perfil utilizado. Nessa mesma tabela, mostram-se ainda os índices discriminantes, onde $\mathrm{R}_{0}$ representa a separação entre os dois litotipos, $\mathrm{R}_{\mathrm{A}}$ é o índice discriminante característico dos arenitos e $R_{B}$ é o índice discriminante característico dos carbonatos. O critério de classificação é o mesmo adotado na classificação anterior a partir do índice discriminante $\mathrm{R}_{0}$.

No caso do Campo de Namorado, os resultados da aplicação das funções discriminantes com os coeficientes definidos nas Tabelas 1 e 3 para o poço de referência, podem ser visualizados na figura 9A, onde é mostrada a identificação dos tipos litológicos

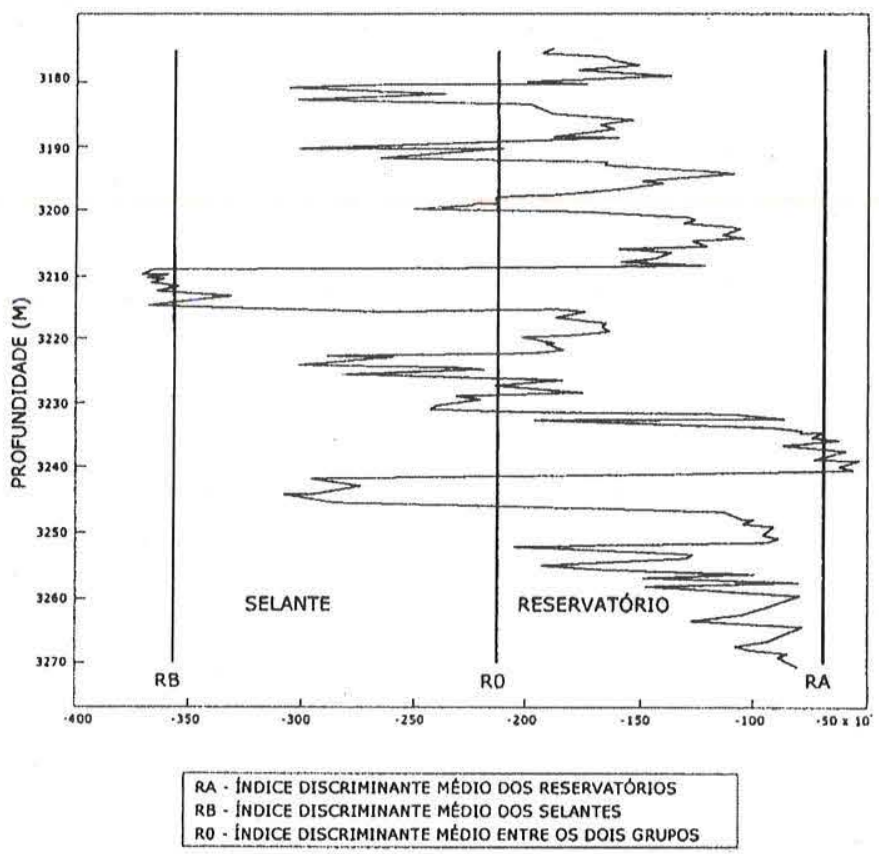

Figura 7-Exemplo do processo discriminatório dos limites entre selantes e reservatórios no Poço NAO2 do Campo de Namorado. em profundidade auxiliada com a apresentação do perfil de raios gama. Observa-se uma boa equivalência entre a interpretação do perfil e a identificação dos tipos litológicos resultantes do processo discriminatório. Na figura 9B, mostra-se um detalhe de um trecho discriminado do poço de referência, que pode ser comparado com a análise seqüencial de testemunho (Fig. 9C). A metodologia da interpretação do poço de referência (NA02) pode ser estendida a outro poço vizinho (NA04), a fim de se verificar a eficiência do método na identificação de litotipos no mesmo campo. Os resultados da aplicação das funções discriminantes com os coeficientes definidos nas Tabelas 1 e 3 constam da figura 10A, onde mostrase a identificação dos tipos litológicos em profundidade auxiliado com a apresentação do perfil de raios gama. Ao igual que os outros casos apresentados, observa-se excelente correspondência entre a interpretação do perfil com a identificação dos tipos

Tabela 1 - Coeficientes da função discriminante utilizada para a identificação dos selantes e reservatórios do Campo de Namorado, Bacia de Campos, Brasil.

\begin{tabular}{c|c|c|c|c|c|c}
\hline \multicolumn{4}{c|}{ Coeficiente discriminante } & \multicolumn{3}{c}{ Índice discriminante $\left(\times 10^{2}\right)$} \\
\hline$G R$ & $D T$ & $\phi_{N}$ & $I L D$ & $R_{0}$ & $R_{A}$ & $R_{B}$ \\
\hline$-37,4$ & 8,4 & $-52,5$ & 0,01 & $-23,9$ & $-41,0$ & $-32,4$ \\
\hline
\end{tabular}

Tabela 2 - Coeficientes da função discriminante utilizada para a identificação dos selantes e reservatórios do Lago Maracaibo, Venezuela.

\begin{tabular}{c|c|c|c|c|c|c}
\hline \multicolumn{4}{c|}{ Coeficiente discriminante } & \multicolumn{3}{|c}{ Índice discriminante $\left(\times 10^{2}\right)$} \\
\hline$G R$ & $\rho_{b}$ & $\phi_{N}$ & $I L D$ & $R_{0}$ & $R_{A}$ & $R_{B}$ \\
\hline$-217,6$ & 124,3 & $-16431,4$ & $-0,3$ & $-212,2$ & $-667,2$ & $-357,2$ \\
\hline
\end{tabular}

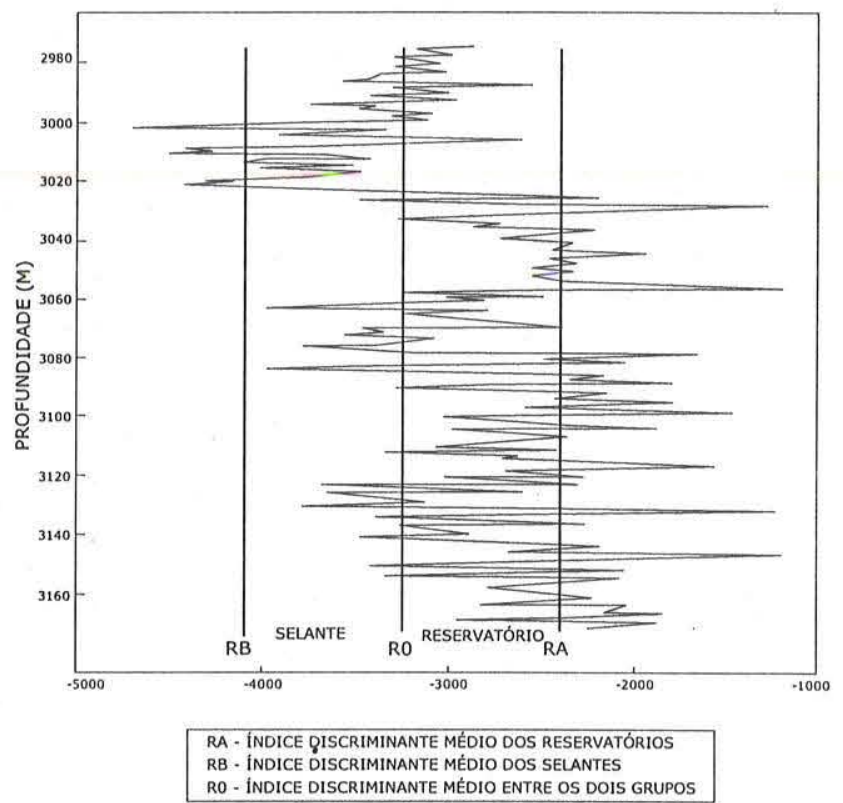

Figura 8-Exemplo do processo discriminatório dos limites entre selantes e reservatórios no Poço 1112 do Lago Maracaibo. 
litológicos. A figura 10B mostra o detalhe de um trecho discriminado do poço NA04, que pode ser comparado com a análise seqüencial de testemunho (Fig. 10C). Observa-se na figura 10B que vários trechos foram discriminados como carbonatos, possivelmente influenciados pelo alto teor de $\mathrm{CaCO}_{3}$ das margas existentes nestes trechos, conforme mostrado na análise seqüencial de testemunho na figura $10 \mathrm{C}$.

Por outro lado, no caso do Lago Maracaibo, os resultados da aplicação das funções discriminantes com os coeficientes definidos na Tabela 2 para o poço de referência, constam da figura 11A, onde é mostrada a identificação dos litotipos em profundidade auxiliada pelo perfil de raios gama. Observa-se também uma boa concordância entre a interpretação do perfil e a identificação dos tipos litológicos resultantes do processo discriminatório, feita por Bryant et al. (1998). A metodologia da interpretação do poço de referência (1112) foi estendida a outro poço vizinho (991), a fim de se verificar a eficiência do método na identificação de tipos litológicos no mesmo campo. Os resultados da aplicação das funções discriminantes com os coeficientes definidos na Tabela 2

Tabela 3 - Coeficientes da função discriminante utilizado para a identificação dos arenitos e carbonatos do Campo de Namorado, Bacia de Campos, Brasil.

\begin{tabular}{c|c|c|c|c|c|c}
\hline \multicolumn{4}{c|}{ Coeficiente discriminante } & \multicolumn{3}{c}{ Índice discriminante $\left(\times 10^{2}\right)$} \\
\hline$G R$ & $D T$ & $\phi_{N}$ & $\rho_{b}$ & $R_{0}$ & $R_{A}$ & $R_{B}$ \\
\hline 28,1 & $-8,4$ & $-6,4$ & $-7465,5$ & $-14,0$ & $-17,2$ & $-15,6$ \\
\hline
\end{tabular}

podem ser visualizados na figura $11 \mathrm{~B}$, onde mostra-se a identificação dos tipos litológicos em profundidade auxiliado com a apresentação do perfil de raios gama. Observa-se excelente concordância entre a interpretação do perfil com a identificação dos tipos litológicos, também realizada por Bryant et al. (1998).

CONCLUSÕES Deve ser ressaltado que aos perfis geofísicos utilizados neste trabalho não se aplicou qualquer préprocessamento, mantendo-se os dados originais. Observa-se, também, que com a utilização das curvas dos perfis pré-processados e da análise discriminante associada a outros mos da estástica multivariada, como análise de agrupamerio. . . inente principal e outros, poder-se-ia ter uma melhoria is processo discriminatório.

Por outro lado, a utilização da análise discriminante aplicada na identificação de litotipo nos perfis de poço apresentou desempenho satisfatório. Os resultados obtidos foram coerentes com a interpretação dos perfis e com os resultados da análise seqüencial de testemunho realizada pela PETROBRAS nos Poços NA02 e NA04 do Campo de Namorado. O mesmo pode-se concluir dos Poços 1112 e 991 do Lago Maracaibo.

Contudo, ressalta-se que mesmo tendo obtido bons resultados na utilização da análise discriminante no caso bivariado para ambos os casos, faz-se necessário implementar programas de análise discriminante para o caso multivariado, capazes de realizar discriminações entre mais de dois grupos, por exemplo, selantes com reservatórios, e dentre destes, diferentes tipos de reservatórios como carbonatos, arenitos, coquinas, basaltos fraturados, turbiditos, etc.

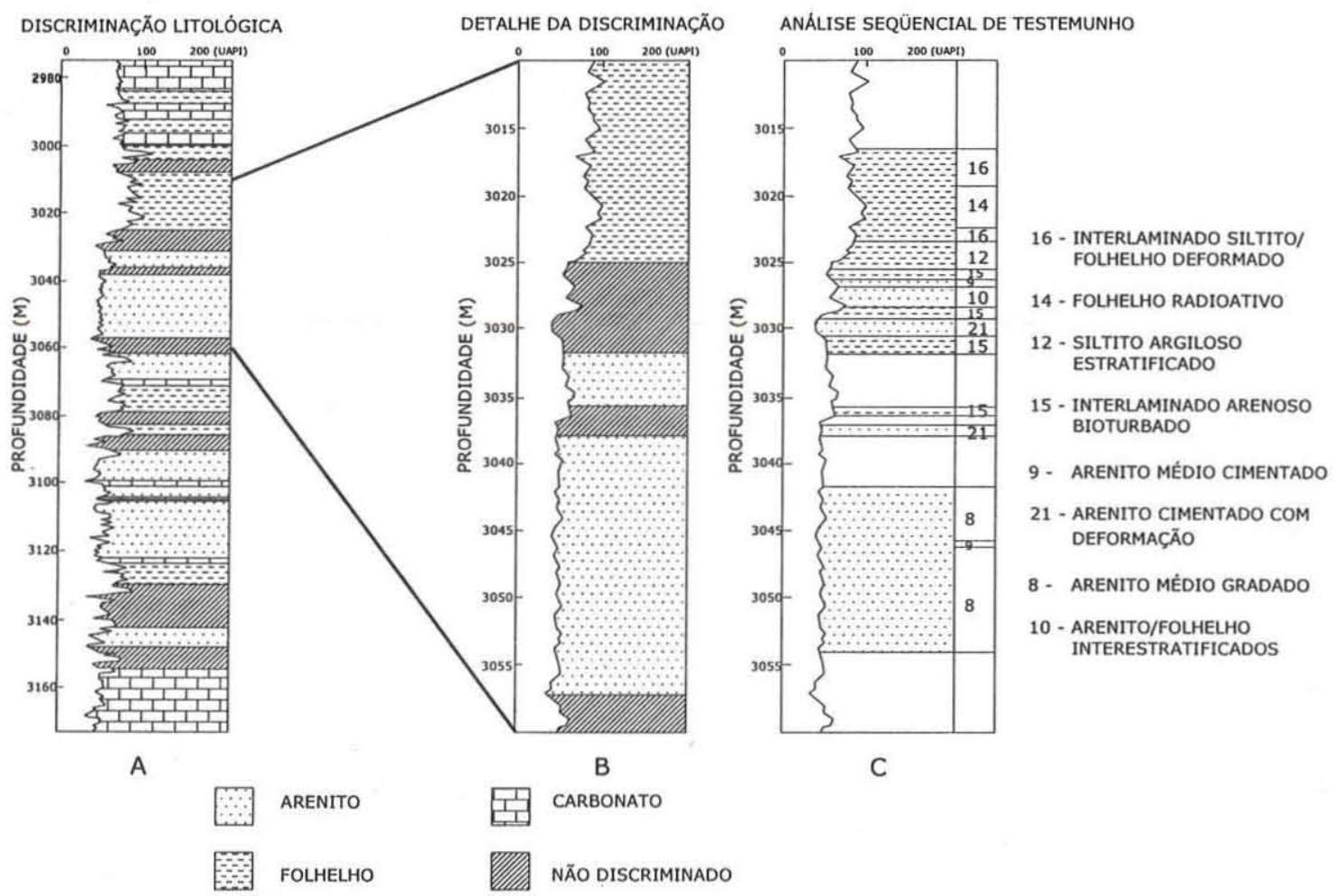

Figura 9-Poço NAO2 do Campo de Namorado. A - Resultado da discriminação litológica, B - Detalhe de um trecho discriminado, $C$-Análise seqüencial de testemunho. 
Agradecimentos À UENF e ao CNPq, pela bolsa de doutorado e produtividade aos autores, a Alfredo Carrasco e Sandra
Matsumura pela revisão do texto e aos revisores da RBG pelas sugestões ao manuscrito.

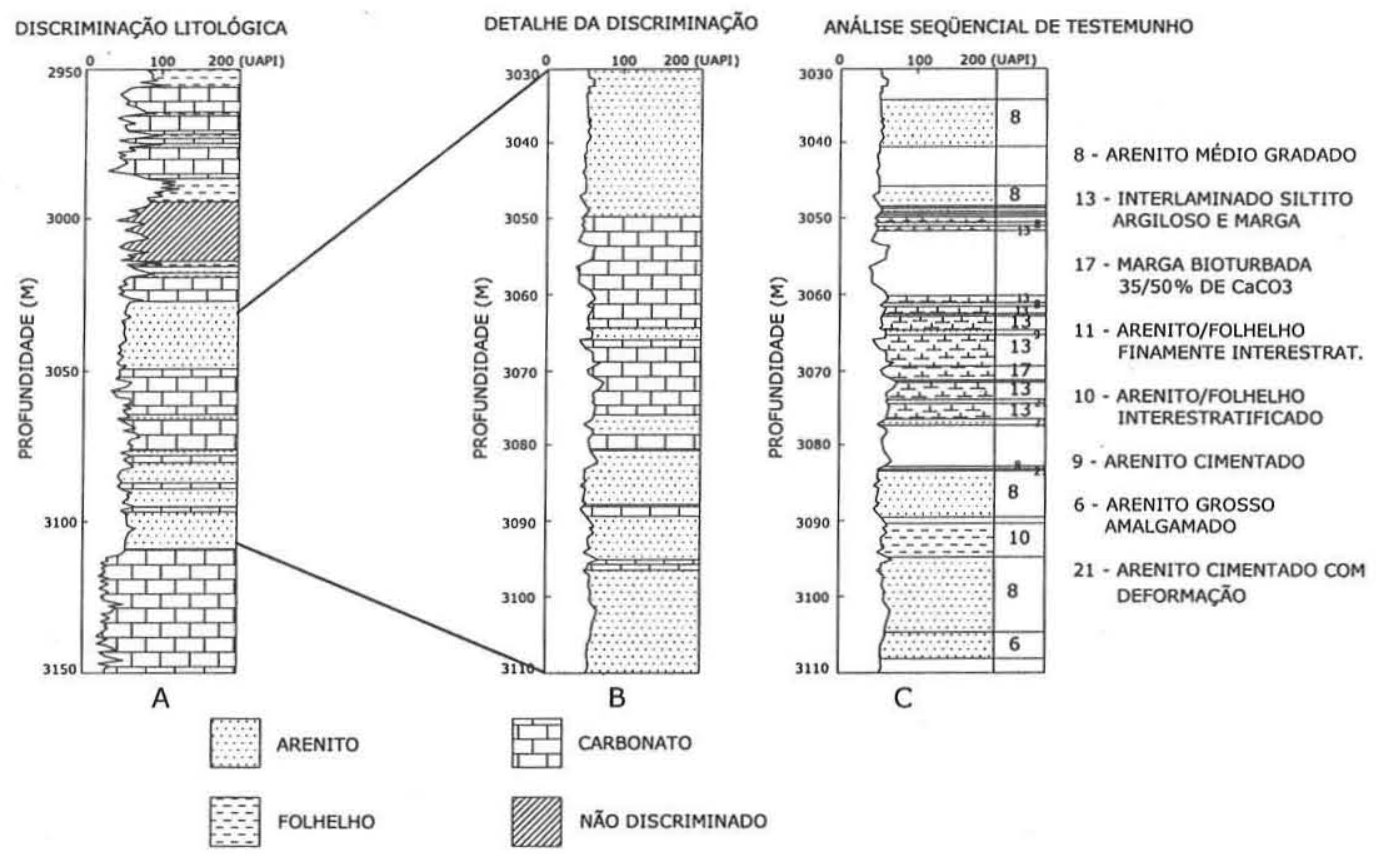

Figura 10 - Poço NA04 do Campo de Namorado. A - Resultado da discriminação litológica, B - Detalhe de um trecho discriminado, $C$ - Análise seqüencial de testemunho.

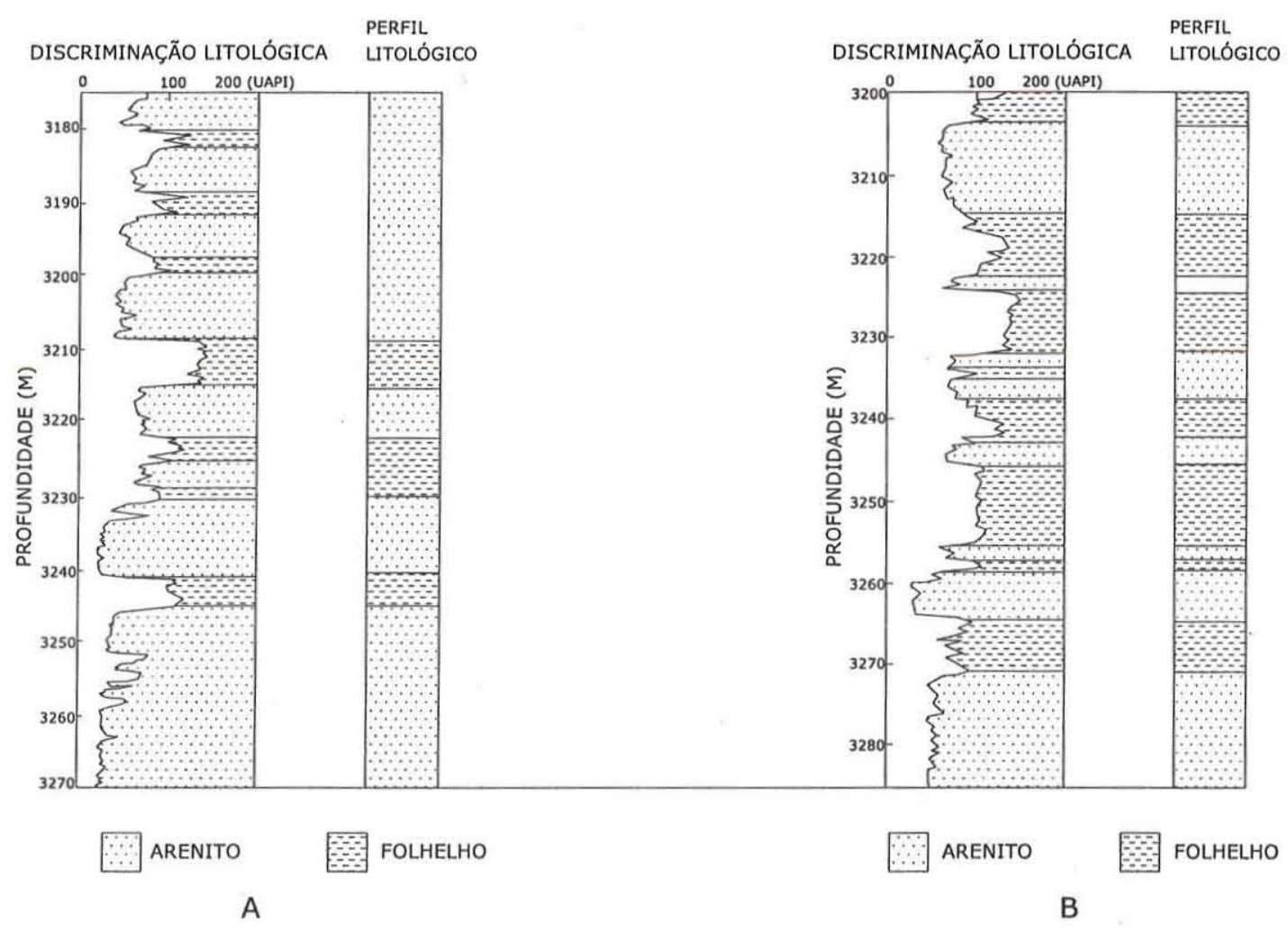

Figura 11 - Resultados da discriminação litológica e análise seqüencial de testemunho feito por Bryant et al. (1998) para os Poços 1112 e 991 do Lago Maracaibo. 
Identificação da litologia nos perfis de poço do campo de Namorado (Bacia de Campos, Brasil) e do lago Maracaibo (Venezuela) aplicando estatística multivariada

\section{Referências}

Bryant I.D., Baygün B., Herron M.M., Matteson A., Ramamoorthy R., Stewart L., Ttariq S.M., Coll C., Córdova P., Villarroel H.G., Hernández L., Jimenez Z., León K., Rondón L. 1998. Integration of old and new measurements to optimize redevelopment of the Lower Lagunillas reservoir of bloque IV, Lake Maracaibo, Venezuela. Society of Petroleum Engineers. SPE Reservoir Evaluation \& Engineering. Paper (SPE 36096).

Bucheb J.A. 1991. Aplicação de tratamento estatístico multivariante em dados de perfis de poços da Bacia de Sergipe, Alagoas. Tese de Mestrado, CG/UFPA, Belém, 136 p.

Couto N.A.P. 1994. Construção de bancos de dados petrofísicos para determinaçâo automática de litologias. Tese de Mestrado, CG/ UFPA, Belém, 113 p.

Davis J.C. 1973. Statistic and data analysis in Geology. New York, John Wiley \& Sons, $550 \mathrm{pp}$.

Dias J.L., Scarton J.C., Guardado L.R., Esteves F.R., Carminatti M. 1990. Aspectos da evolução tectono-sedimentar e a ocorrência de hidrocarbonetos na Bacia de Campos. In: G.P. Raja Gabaglia \& E.J. Milani (eds.) Origem e evolução de bacias sedimentares. Rio de Janeiro, PETROBRAS, pp. 333-360.

Flexa R.T. 200I. Determinação estatística dos contatos fluidos em perfis de poço. Tese de Mestrado, CG/UFPA, Belém, 29 p.

Guardado L.R., Spadini A.R, Brandão J.S.L., Mello M.R. 2000. Petroleum system of the Campos Basin, In: M.R. Mello \& B.J. Katz (eds.) Petroleum systems of South Atlantic margins: AAPG Memoir 73, p. $317-324$

Nitters G., Davies D.R., Epping W.J.M. 1995. Discriminant analysis and neural nets: Valuable tools to optimize completion practices, SPE Drilling \& Completion, p. 127-133.

Rangel H.D., Martins F.A.L., Esteves F.R., Feijó F.J. 1994. Bacia de Campos, Boletim de Geociências da PETROBRAS, Rio de Janeiro, 8(1):203-217.

Serra O. \& Abbot H. T. 1989. The contribution of logging data to sedimentology and stratigraphy, SPE 9270, 18 p.

Talukdar S.C. \& Maracano F. 1994. Petroleum systems of the Maracaibo Basin, Venezuela. The petroleum system - from source to trap. AAPG Memoir 60.

Manuscrito A-1449

Recebido em 09 de julho de 2003

Revisão dos autores em 30 de maio de 2004 Revisão aceita em 15 de junho de 2004 